\title{
VENTILAÇÃO PULMONAR E SUA RELAÇÃO COM MEDIDAS DO QUOCIENTE RESPIRATÓRIO E PERCENTUAL DE GORDURA: ESTUDO PRELIMINAR
}

\section{RESUMO}

\author{
Marcos Vinícios Jacobs ${ }^{1}$ \\ Éboni Marília Reuter ${ }^{2}$ \\ Valeriano Antonio Corbellini $i^{3}$ \\ Miriam Beatrís Reckziegel ${ }^{4}$ \\ Hildegard Hedwig Poht ${ }^{5}$
}

A ventilação pulmonar depende da interação dos compartimentos torácico e abdominal, havendo incremento de trabalho ventilatório na presença de acúmulo de gordura nesta região corporal. O objetivo foi avaliar a relação da ventilação pulmonar com marcadores do quociente respiratório $(\mathrm{QR})$ e o percentual de gordura $(\% \mathrm{G})$ em trabalhadores da agroindústria e produtores rurais. Com delineamento transversal, foram avaliados dezoito trabalhadores do município de Santa Cruz do Sul - RS, sendo selecionados dados de \%G e do teste ergoespirométrico (protocolo de Bruce), obtendo-se resultados diretos do consumo de oxigênio $\left(\mathrm{VO}_{2}\right)$, dióxido de carbono produzido $\left(\mathrm{VCO}_{2}\right)$ e ventilação pulmonar (VE). Os resultados indicam associação regular entre $\mathrm{VE}$ com as variáveis de $\mathrm{VO}_{2}(\mathrm{r}=0,564$ e $\mathrm{p}=$ $0,015)$ e \%G ( $\mathrm{r}=-0,540$ e $\mathrm{p}=0,021)$. Já entre a $\mathrm{VE}$ e o $\mathrm{VCO}_{2}$ foi evidenciada associação forte $(\mathrm{r}=0,775$ e $\mathrm{p}<0,001)$. A associação inversa entre $\mathrm{VE}$ e $\% \mathrm{G}$ sugere a redução da capacidade ventilatória quando há aumento da massa gorda, o que pode ser em consequência da restrição mecânica da caixa torácica adjunto à baixa capacidade de trabalho dos músculos ou tolerância aos exercícios físicos.

Palavras-chave: Trabalhadores Rurais. Ventilação Pulmonar. Obesidade.

\begin{abstract}
Lung ventilation depends on the interaction of thoracic and abdominal compartments, with increment in ventilation work in the presence of body fat accumulation in this region. The objective was to evaluate the correlation of lung ventilation with markers of respiratory quotient and body fat percentage $(\mathrm{BF} \%)$ in agribusiness workers and rural producers. Through a cross-sectional design, eighteen workers from Santa Cruz do Sul - RS, were evaluated, selecting data of $\mathrm{BF} \%$ and cardiopulmonary exercise test (Bruce protocol), obtaining direct results of oxygen consumption $\left(\mathrm{VO}_{2}\right)$, carbon dioxide produced $\left(\mathrm{VCO}_{2}\right)$ and lung ventilation (LV). Results indicate regular association between $\mathrm{LV}$ with variables of $\mathrm{VO}_{2}(\mathrm{r}=0.564$ and $\mathrm{p}$ $=0.015)$ and $\mathrm{BF} \%(\mathrm{r}=-0.540$ and $\mathrm{p}=0.021)$. However, between $\mathrm{LV}$ and $\mathrm{VCO}_{2}$ there was evidence of strong association $(r=0.775$ and $p<0.001)$. The inverse association between LV and $\mathrm{BF} \%$ suggests a reduction in ventilatory capacity when there is increased fat mass, which may be a result of mechanical restriction of chest adjunct to low work capacity of muscles or exercise tolerance.
\end{abstract}

Keywords: Rural Workers. Pulmonary Ventilation. Obesity.

\footnotetext{
${ }^{1}$ Aluno de Educação Física pela Universidade de Santa Cruz do Sul - UNISC. <mv jacobs@ hotmail.com>

${ }^{2}$ Mestranda em Promoção da Saúde pela Universidade de Santa Cruz do Sul - UNISC. <ebonir@ outlook.com>

${ }^{3}$ Professor do Departamento de Química e Física - Universidade de Santa Cruz do Sul. <valer@unisc.br>

${ }^{4}$ Professora do Departamento de Educação Física e Saúde da Universidade de Santa Cruz do Sul - UNISC. <miriam@unisc.br>

${ }^{5}$ Professora do Departamento de Educação Física e Saúde da Universidade de Santa Cruz do Sul - UNISC. 〈hpohl@unisc.br>
} 


\section{INTRODUÇÃO}

A ventilação pulmonar depende da interação dos compartimentos torácico e abdominal, na medida em que no processo respiratório é necessário o recrutamento de algumas estruturas anatômicas que compõem a parede torácica, como os tecidos responsáveis pela realização do ciclo ventilatório de inspiração e expiração (PRESTO; PRESTO, 2005; SARRO, 2007). Neste sentido, a presença da obesidade e, principalmente, o acúmulo de gordura corporal nesta região leva ao incremento do trabalho ventilatório, podendo comprometer as funções pulmonares e respiratórias (AGUIAR et al., 2012).

Em decorrência do excesso de gordura corporal, principalmente nas regiões torácicas e abdominais, as funções pulmonares são comprometidas e, na maioria dos casos, ocorre uma queda na capacidade respiratória, com reflexo no processo de ventilação/perfusão, sendo que as dificuldades respiratórias variam conforme o grau de adiposidade corporal (SCIPIONI, 2010; TEIXEIRA et al., 2007; PINHEIRO; FREITAS; CORSO, 2004). A obesidade exerce uma relevante carga no sistema respiratório, de forma a provocar modificações tanto nos volumes respiratórios como no padrão de respiração, podendo atingir os músculos das vias áreas. Com a presença de uma condição inflamatória sistêmica, originada de moléculas próinflamatórias do tecido adiposo, as alterações podem causar restrição pulmonar e mortalidade cardiovascular (LEITE, 2009).

A redução de volume pulmonar ocorre devido à existência de uma limitação da expansão torácica durante os movimentos respiratórios, ou seja, há uma redução do espaço anatômico com a ocupação de parte da área corpórea com massa gordurosa e isso gera uma sobrecarga nos músculos envolvidos no processo de ventilação pulmonar (AGUIAR et al., 2012; COSTA et al., 2003; FRANCISCHI et al., 2000). A presença de massa gorda, em grandes proporções, afeta também o balanço estático do sistema respiratório, o qual fica comprometido devido às alterações causadas dentro do tórax e abdômen (OLIVEIRA et al., 2006).

Diante das premissas expostas, este estudo teve como objetivo avaliar a relação da ventilação pulmonar com marcadores do quociente respiratório e o percentual de gordura em trabalhadores da agroindústria e produtores rurais. 


\section{FUNDAMENTAÇÃO TEÓRICA}

O sistema respiratório tem como função principal o fornecimento de oxigênio e a eliminação de dióxido de carbono das células corpóreas. Para ocorrência desse processo, necessita-se recrutar algumas estruturas anatômicas que compõem a parede torácica, como os tecidos responsáveis pela realização do ciclo ventilatório de inspiração e expiração (PRESTO; PRESTO, 2005; SARRO, 2007). Os movimentos respiratórios ocorrem ininterruptamente, promovendo mudanças na caixa torácica e no abdômen e, consequentemente, alterações na geometria da cavidade torácica (NAGATO et al., 2012; RECIERI, 2001; SILVA; SILVA, 2006).

Um dos parâmetros do sistema respiratório avaliados é a ventilação pulmonar (VE), que se caracteriza como a quantidade de ar que entre e sai dos pulmões, expresso em litros por minuto (L/min.). A VE compreende a soma dos gases de ventilação alveolar (Va) e do espaço morto (Vd) (BARROS NETO; TEBEXRENI; TAMBEIRO, 2001).

As disfunções respiratórias podem estar relacionadas ao elevado índice de massa gorda nas regiões peritorácica e abdominal e, consequentemente, influenciar na mecânica respiratória (FRANÇA, 2004; OLIVEIRA et al., 2006; SALVE, 2006; SCIPIONI, 2010, TEIXEIRA et al., 2007). Nesta condição, do ponto de vista muscular, o diafragma acaba por permanecer elevado em decorrência da distensão do abdômen. A partir da necessidade dos músculos intercostais moverem uma grande quantidade de massa, esta condição física dificulta o trabalho mecânico de respiração (AGUIAR et al., 2012; COSTA et al., 2003; NAGATO et al., 2012). Essa modificação está associada à retração elástica e diminuição da distensibilidade das estruturas que compreendem a parte externa do sistema pulmonar (RASSLAN et al., 2004; RIBEIRO et al., 2007).

A obesidade se caracteriza pelo acúmulo de tecido adiposo acima do recomendado e é considerada uma doença crônica não transmissível (BOSON; GARDENGHI, 2012; GUIMARÃES; MARTINS; SANTOS, 2012; HEYWARD, 2004; SALVE, 2006). As disfunções cardiometabólicas dos obesos, que afetam vários tecidos e/ou sistemas corporais, tornam os sujeitos mais suscetíveis ao desenvolvimento de doenças, como os problemas pulmonares (FRANÇA, 2004; OLIVEIRA et al., 2006; SALVE, 2006; SCIPIONI, 2010, TEIXEIRA et al., 2007).

Atualmente a obesidade é considerada uma epidemia mundial, sendo que conforme os índices da Organização Mundial de Saúde (2012), 12\% da população mundial é obesa. A alteração do perfil antropométrico das pessoas está associada aos processos evolutivos, sendo 
estes de caráter demográfico, socioeconômico e epidemiológico (FANCISCHI et al., 2000; SILVA; SILVA, 2006). Desta forma, os hábitos de vida foram influenciados negativamente, aumentando os índices de sujeitos insuficientemente ativos e do consumo de alimentos com elevado valor calórico (SALVE, 2006).

\section{MATERIAIS E MÉTODOS}

Este artigo caracteriza-se pelo estudo de delineamento transversal, tendo sido avaliados dezoito trabalhadores da agroindústria e produtores rurais do município de Santa Cruz do Sul/RS, pertencente à microrregião Centro do Vale do Rio Pardo (VRP) (SILVEIRA; CAMPOS, 2012). O grupo de trabalhadores analisado neste artigo é composto por integrantes de um projeto de pesquisa que assinaram o Termo de Consentimento Livre e Esclarecido quando convidados a participar do estudo (projeto aprovado pelo CEP-UNISC protocolo 2509/10)

Caracterizam-se tanto a cidade quanto a região com uma atividade econômica centrada na agroindústria, sendo que neste estudo foram priorizados trabalhadores das atividades hortigranjeiros. Foram avaliados trabalhadores rurais, incluindo aqueles que trabalham com a produção, bem como aqueles que beneficiam a matéria-prima (agroindústria) (SILVEIRA; CAMPOS, 2012). A coleta de dados ocorreu em duas etapas, constando a primeira da seleção dos participantes através de um questionário de estilo de vida e estimativa do índice de massa corporal (IMC) (POHL et al., 2010). A partir do questionário foram selecionadas variáveis demográficas (idade e sexo), além da avaliação econômica pelos Critérios de Classificação Econômica do Brasil, proposto pela Associação Brasileira de Empresas de Pesquisa (ABEP, 2012).

$\mathrm{Na}$ segunda fase, os sujeitos selecionados foram submetidos às avaliações clínicas e funcionais, que constaram de antropometria, análises bioquímicas e teste cardiorrespiratório, no qual foca-se o estudo em valores do teste ergoespirométrico e medida antropométrica de gordura total. Na avaliação antropométrica foram mensuradas as dobras cutâneas para estimativa do percentual de gordura $(\% \mathrm{G})$, utilizando o compasso de Lange e as diretrizes Heyward (2004), em que se consideraram sete dobras cutâneas (DC). A soma das DC foi acrescida na equação de Jackson e Pollock para cálculo da densidade corporal e, posteriormente, de Siri, sendo o resultado final classificado segundo Pollock e Wilmore (1993). 
$\mathrm{Na}$ avaliação cardiorrespiratória os trabalhadores foram submetidos a um teste de esforço submáximo pelo protocolo de Bruce modificado (Heyward, 2004), numa esteira ergométrica acoplada ao analisador de gases VO2000. Neste teste foi possível monitorar e registrar o consumo de oxigênio $\left(\mathrm{VO}_{2}\right)$, produção de dióxido de carbono $\left(\mathrm{VCO}_{2}\right)$ e a ventilação pulmonar (VE), sendo que para análise estatística se levou em conta o valor de pico.

Todos os dados coletados nas avaliações foram anexados a uma planilha eletrônica, sendo eles categorizados e analisados no software Statistical Package for Social Sciences for Windows (SPSS - versão 20.0). Os valores numéricos foram dispostos em percentis, média e desvio padrão e os categóricos em frequência e percentual para descrição da amostra. Já a fase analítica partiu da verificação da normalidade pelo teste de Shapiro-Wilk, e, posteriormente, realizada as correlações através do teste de Pearson, considerando nível de significância $\mathrm{p}<$ 0,05. A avaliação qualitativa das correlações seguiu as categorias propostas por CallegariJacques (2003).

\section{RESULTADOS E DISCUSSÃO}

Os resultados da pesquisa caracterizam trabalhadores da agroindústria e produtores rurais, de ambos os sexos, idade média de 51,44 $( \pm 9,88)$, sendo $55,5 \%$ pertencentes às classes econômicas B2 e C1.

Na Tabela 1 constam as variáveis numéricas dos parâmetros estudados. Observa-se similaridade dos dados de tendência central, uma vez que os valores da média e percentil 50 se assemelham em todas as variáveis. Na variável VE o valor máximo encontrado foi de 55,60 $\mathrm{L} / \mathrm{min}$, enquanto que $\% \mathrm{G}$ variou entre $17,58 \%$ a $33,82 \%$.

Tabela 1 - Apresentação das medidas de tendência central e dispersão das medidas de $\mathrm{VE}, \mathrm{VO}_{2}, \mathrm{VCO}_{2} \mathrm{e} \% \mathrm{G}$.

\begin{tabular}{ccccc}
\hline & VE & $\mathbf{V O}_{\mathbf{2}}$ & $\mathbf{V C O}_{\mathbf{2}}$ & \%G \\
\hline Média & 38,76 & 20,47 & 23,80 & 25,64 \\
Percentil 25 & 30,88 & 16,39 & 19,37 & 20,92 \\
Percentil 50 & 37,80 & 20,23 & 22,64 & 26,66 \\
Percentil 75 & 48,12 & 23,34 & 26,69 & 30,13 \\
DP & 9,47 & 5,14 & 5,25 & 5,03 \\
\hline
\end{tabular}

VE: Ventilação pulmonar; $\mathrm{VO}_{2}$ : Consumo de oxigênio; $\mathrm{VCO}_{2}$ : Produção de dióxido de carbono; \%G: Percentual de gordura; DP: Desvio padrão. 
Os resultados categóricos do \%G se apresentaram favoráveis à saúde uma vez que $77,8 \%$ dos sujeitos foram classificados com resultados positivos $(11,1 \%$ na média; $50,0 \%$ acima da média; $16,7 \%$ bom) e 22,2\% foram categorizados como abaixo da média. Não houve ocorrência de sujeitos com \%G excelente, ruim ou muito ruim. Neste estudo não foi considerada a distribuição da gordura corporal, entretanto sabe-se que o acúmulo localizado na região abdominal oferece risco à saúde, pois pode predispor o desenvolvimento de distúrbios metabólicos e complicações cardiovasculares (BOUCHARD, 2003; FRANÇA, 2004; FRANCISCHI, 2000; SALVE, 2006; WANG; HOY, 2004).

Analisando as correlações entre variáveis cardiorrespiratórias, apresentadas na Tabela 2, observam-se associações regulares entre VE com as variáveis de $\mathrm{VO}_{2}$ e \%G ( $\mathrm{r}=0,564$ e $\mathrm{p}=0,015 ; \mathrm{r}=-0,540$ e $\mathrm{p}=0,021$, respectivamente). Também foi evidenciada uma associação forte $(\mathrm{r}=0,775$ e $\mathrm{p}<0,001)$ entre a $\mathrm{VE}$ e o $\mathrm{VCO}_{2}$.

Tabela 2 - Correlação da VE com $\mathrm{VO}_{2}, \mathrm{VCO}_{2}$ e \%G.

\begin{tabular}{ccccc}
\hline Variáveis & & $\mathbf{V O}_{2}$ & $\mathbf{V C O}_{2}$ & \% G \\
\hline \multirow{2}{*}{ VE } & $\mathbf{P}$ & $0,564^{* *}$ & $0,775^{*}$ & $-0,540^{* *}$ \\
& $\mathbf{R}$ & 0,015 & 0,021 & $<0,001$ \\
\hline
\end{tabular}

* Correlação forte $(r=0,6$ a 0,9); ** Correlação regular $(r=0,3$ a 0,6); r: Correlação linear de Pearson; p: Nível de significância; VE: Ventilação pulmonar; $\mathrm{VO}_{2}$ : Consumo de oxigênio; $\mathrm{VCO}_{2}$ : Produção de dióxido de carbono; \%G: Percentual de gordura.

A associação negativa entre o \%G com a VE pode ser relacionada a redução da porcentagem de massa gorda e tende a contribuir para elevação da capacidade de ventilação pulmonar (LEITE, 2009; SCIPIONI, 2010; TEIXEIRA et al., 2007). Com aumento do \%G, principalmente na cavidade abdominal e torácica, o diafragma permanece elevado em decorrência da distensão do abdômen e, a partir da necessidade dos músculos intercostais moverem uma grande quantidade de massa, esta condição física dificulta o trabalho mecânico de respiração (AGUIAR et al., 2012; COSTA et al., 2003; NAGATO et al., 2012).

Em relação às correlações das medidas de $\mathrm{VO}_{2}$ e $\mathrm{VCO}_{2}$ com a $\mathrm{VE}$, percebe-se que ambas foram lineares, sendo categorizadas com grau forte. Essas respostas condizem com a literatura científica, pois o aumento do $\mathrm{VO}_{2}$ e $\mathrm{VCO}_{2}$ implica na maior ventilação de gases, exceto em pessoas portadoras de morbidades respiratórias (BLUMENKRANTZ, 1997; PRESTO; PRESTO, 2005).

Diversos estudos têm enfocado a relação entre obesidade e a restrição da mecânica respiratória com prejuízos no trabalho respiratório. Indicam também a redução da capacidade ventilatória em indivíduos com elevados níveis de gordura corporal (AGUIAR et al., 2012; BOSON; GARDENGHI, 2012; COSTA et al., 2003; GUIMARÃES; MARTINS; SANTOS, 
2012; RASSLAN et al., 2004). Cabe ressaltar que estes estudos avaliaram a função pulmonar utilizando a espirometria e com o avaliado em repouso, enquanto que o trabalho analisou a capacidade cardiorrespiratória através do teste ergoespirométrico, considerando as medidas respiratórias diretas do analisador de gases VO2000. Entende-se que esta diferença metodológica restringe maiores comparações entre os resultados de diferentes estudos (LEITE, 2009; PRESTO; PRESTO, 2005; RIBEIRO et al., 2007).

Observaram-se associações regulares entre $\mathrm{VE}$ com as variáveis de $\mathrm{VO}_{2}$ e \%G, enquanto que entre a $\mathrm{VE}$ e o $\mathrm{VCO}_{2}$ foi evidenciada uma correlação forte, indicando dependência entre elas, ou seja, as alterações presentes numa medida estão presentes na outra. A associação inversa entre VE e \%G sugere a redução da capacidade ventilatória quando há aumento da massa gorda, o que pode ser decorrente da restrição mecânica da caixa torácica adjunto à baixa capacidade de trabalho dos músculos ou tolerância aos exercícios físicos. Vale ressaltar que as complicações cardiometabólicas apresentadas por alguns avaliados, podem estar associadas ao estilo de vida dos mesmos, além de fatores intrínsecos, como faixa etária, etnia, sexo. Entende-se ainda que com o acesso das pessoas às informações de educação em saúde, tende-se a diminuir a presença de sujeitos com risco de comorbidades.

\section{AGRADECIMENTOS}

À Universidade de Santa Cruz do Sul - UNISC, à Secretaria de Ciência, Inovação e Desenvolvimento Tecnológico do Estado do Rio Grande do Sul (SCIDT/RS), ao Polo de Modernização Tecnológica do Vale do Rio Pardo, à Fundação de Amparo à Pesquisa do Estado do Rio Grande do Sul (FAPERGS/RS), ao Conselho Nacional de Desenvolvimento Científico e Tecnológico (CNPq) e ao Programa de Pós-Graduação em Promoção da Saúde (PPGPS/UNISC).

\section{REFERÊNCIAS}

AGUIAR, I. C. et al. Capacidade pulmonar e força ventilatória em obesos mórbidos. Revista Terapia Manual, v. 10, n. 47, p. 71-74, 2012.

ASSOCIAÇÃO BRASILEIRA DE EMPRESAS DE PESQUISA (ABEP). Dados com base no levantamento sócio econômico 2011 - IBOPE. 2012; Disponível em: <www.abep.org>. Acesso em: 06 jan. 2014. 
BARROS NETO, T. L.; TEBEXRENI, A. S.; TAMBEIRO, V. L. Aplicações práticas da ergoespirometria no atleta. Revista da Sociedade de Cardiologia do Estado de São Paulo. São Paulo, v. 11, n. 3 p. 695-705, 2001.

BLUMENKRANTZ, Michael. Obesity: the world's metabolic disorder. Beverly Hills, 1997. Disponível em: <http:// www.quantumhcp.com,obesity.htm >. Acesso em: 24 jan. 2014.

BOSON, N. L. B.; GARDENGHI, G. Estudo de pico do fluxo expiratório em obesos candidatos à cirurgia bariátrica. Revista Eletrônica Saúde e Ciência, v. 2, n. 2, p.19-29, 2012.

BOUCHARD, C. Atividade física e obesidade. São Paulo: Manole, 2003.

CALLEGARI-JACQUES, Sidia Maria. Bioestatística: princípios e aplicações. Porto Alegre: Artmed; 2003.

COSTA, D. et al. Avaliação da força muscular respiratória e amplitudes torácicas e abdominais após a RFR em indivíduos obesos. Revista Latino Americana de Enfermagem, Ribeirão Preto, v. 11, n. 2, p.1-9, 2003.

FRANCISCHI, R. P. P. et al. Obesidade: atualização sobre sua etiologia, morbidade e tratamento. Revista de Nutrição, Campinas, v. 13, n. 1, p. 17-28, 2000.

FRANÇA, A. A. Obesidade e a repercussão na Saúde. 2004. 56 f. Projeto de pesquisa (Curso de Especialização em Qualidade de Alimentos - Especialização) - Universidade de Brasília, Brasília, 2004.

GUIMARÃES, C.; MARTINS, M. V.; SANTOS, M. Função pulmonar em doentes obesos submetidos à cirurgia bariátrica. Revista Portuguesa de Pneumologia, v. 18, n. 3, p. 115-119, 2012.

HEYWARD, V. H. Avaliação física e prescrição de exercício: técnicas avançadas. 4. ed. Porto Alegre: Artmed, 2004.

LEITE, J. M. Obesidade infantil e alterações das provas funcionais respiratórias. 2009. $50 \mathrm{f}$. Dissertação (Mestrado Integrado em Medicina - Faculdade de Ciências da Saúde) Universidade da Beira Interior, Covilhã, 2009.

NAGATO, A. C. et al. Influência do posicionamento dos membros superiores sobre parâmetros ventilatórios em indivíduos adultos. Fisioterapia e Movimento, v. 25, n. 3, p. 525$532,2012$.

OLIVEIRA, F. B. et al. Análise do efeito da obesidade sobre as propriedades resistivas e elásticas do sistema respiratório por oscilações forçadas. Pulmão, Rio de Janeiro, v. 15, n. 4, p. 219-223, 2006.

ORGANIZAÇÃO MUNDIAL DE SAÚDE (OMS). Relatório de "Estatísticas Mundiais de Saúde 2012”. Disponível em: <http://www.who.int/es/>. Acesso em: 24 ago. 2013.

PINHEIRO, A. R. O.; FREITAS, S. F. T.; CORSO, A. C. T. Uma abordagem epidemiológica da obesidade. Revista de Nutrição, Campinas, v. 17, n. 4, p. 523-533, 2004. 
POLLOCK, M.; WILMORE, J. H. Exercícios na saúde e na doença: avaliação e prescrição para prevenção e reabilitação. 2 ed. Rio de Janeiro: Medsi, 1993.

POHL, H. H., RECKZIEGEL, M. B., VITIELLO, I. P.; GALLIANO, L. M. Worker's health and lifestyle: a multi-sectorial view of the physical fitness. The FIEP Bulletin, Foz do Iguaçu, v. 80, p. 808-812, 2010.

PRESTO, B.; PRESTO, L. B. N. Fisioterapia respiratória: uma nova visão. 2 ed. Rio de Janeiro: Livraria Roberto Palmieri, 2005.

RASSLAN, Z. et al. Avaliação da função pulmonar na obesidade grau I e II. Jornal Brasileiro de Pneumologia, v. 30, n. 6, p. 508-514, 2004.

RECIERI, D. da V. Anatomia da parede torácica e suas relações com os movimentos respiratórios: uma abordagem fisioterápica. Fisio \& Terapia, n. 29, p. 19-16, 2001.

RIBEIRO, G. F. et al. Avaliação da função pulmonar em indivíduos obesos assintomáticos respiratórios: correlação entre dados antropométricos e espirométricos. Revista Brasileira de Alergia e Imunopatologia, v. 30, n. 6, p. 227-231, 2007.

SALVE, M. G. C. Obesidade e peso corporal: riscos e consequências. Movimento \& Percepção, Espírito Santo de Pinhal, v. 6, n. 8, p. 29-48, 2006.

SARRO, K. J. Proposição, validação e aplicação de um novo método para a análise cinemática tridimensional da movimentação da caixa torácica durante a respiração. 2007. 72 f. Tese (Programa de Pós-Graduação em Educação Física) - Universidade Estadual de Campinas, Campinas, 2007.

SCIPIONI, G. Função pulmonar e perda de peso em indivíduos obesos mórbidos submetidos à gastroplastia. 2010. $87 \mathrm{f}$. Dissertação (Setor de Ciências Biológicas da Universidade do Paraná - Curso de Mestrado em Educação Física) - Universidade Federal do Paraná, Curitiba, 2010.

SILVA, I. P.; SILVA; R. Q. Interferência postural no grau de força dos músculos respiratórios na obesidade. 2006. 59 f. Trabalho de conclusão de curso (Centro de Ciências Biológicas e da Saúde - Curso de Fisioterapia) - Universidade da Amazônia (UNAMA), Belém, 2006.

SILVEIRA, R. L. L.; CAMPOS, H. A. Processos participativos em experiências recentes de planejamento regional: o caso do Vale do Rio Pardo (RS). Redes, Santa Cruz do Sul, v. 17, n. 1, p. 203-216, 2012.

TEIXEIRA, C. A. et al. Prevalência de dispneia e possíveis mecanismos fisiopatológicos envolvidos em indivíduos com obesidade graus 2 e 3. Jornal Brasileiro de Pneumologia, v. 33, n. 1, p. 28-35, 2007.

WANG, Z.; HOY, W. E. Waist circumference, body mass index, hip circumference and waist-to-hip ratio as predictors of cardiovascular disease in Aboriginal people. European Journal of Clinical Nutrition, v. 58, p. 888-893, 2004. 